\title{
Incidence of Cushing's syndrome in patients with significant hypercortisoluria
}

\section{Dania Hirsch 1,2,3, Gloria Tsvetov 1,2,3, Yossi Manisterski ${ }^{2,3}$, Nirit Aviran-Barak ${ }^{3}$, Varda Nadler ${ }^{4}$, Sandra Alboim ${ }^{4}$ and Vered Kopel ${ }^{4}$}

${ }^{1}$ Institute of Endocrinology, Rabin Medical Center-Beilinson Hospital, Petach Tikva, Israel, ${ }^{2}$ Sackler Faculty of Medicine, Tel Aviv University, Tel Aviv, Israel, ${ }^{3}$ Maccabi Health Care Services, and ${ }^{4}$ Central Laboratory, Maccabi Healthcare Services, Rehovot, Israel
Correspondence should be addressed to D Hirsch

Email

daniaron@netvision.net.il

\begin{abstract}
Objective: To investigate the incidence of Cushing's syndrome (CS) in patients with significant hypercortisoluria and the performance of urinary free cortisol (UFC) screening.

Design: Retrospective file review.

Methods: The computerized database of a publicly funded health maintenance organization (HMO) in Israel was searched for all patients who underwent 24-h UFC testing in 2005-2014 with a result of more than twice the upper limit of normal (ULN). The patients' medical files were reviewed for a subsequent diagnosis of CS by an expert endocrinologist. Findings were evaluated for patterns in CS diagnosis and UFC testing over time.

Results: Of 41183 individuals tested, 510 (1.2\%) had UFC >2x ULN (214 >3x ULN). Eighty-five (16.7\%) individuals were diagnosed with CS (63 female and mean age $47.2 \pm 15.1$ years), mainly Cushing's disease (55.3\%) or adrenal Cushing's syndrome (37.6\%). The number of UFC tests increased steadily, from 1804 in 2005 to 6464 in 2014 ; yet, the resultant detection rate of CS remained generally stable. The calculated incidence of CS in the general HMO-insured population based only on the patients identified in the present cohort was 4.5 new cases/million/year (median 4.9/million/year, range 1.7-5.9/million/year), which was also relatively stable. The most common reason for referral for UFC screening was obesity. Of the 148 patients before bariatric surgery with UFC $>2 \times$ ULN, 2 were diagnosed with CS.

Conclusions: The incidence of CS is higher than previously suggested. The consistently increasing number of UFC tests being performed has not been accompanied by a similar increase in CS detection rate. The expected yield of routine UFC testing before bariatric surgery is low.

European Journal of

Endocrinology

(2017) 176, 41-48
\end{abstract}

\section{Introduction}

Endogenous pathologic hypercortisolism, or endogenous Cushing's syndrome (CS), is a rare endocrine disorder. Although CS has been extensively investigated, its reported incidence of $0.7-2.4$ per million population per year $(1,2,3)$ is derived from only two historical general population-based studies $(2,3)$. The more recent one collected information on all patients in Denmark diagnosed with CS over an 11-year period (1985-1995). A total of 166 patients were identified for an incidence of 2 cases per million inhabitants per year (2). Of these, 99 (59.6\%) had pituitary-dependent hypercorticism (Cushing's disease). The other study included only the
() 2017 European Society of Endocrinology Printed in Great Britain
49 patients with Cushing's disease living in a single city (Vizcaya, Spain) between 1975 and 1992, which yielded an average incidence of 2.4 cases per million inhabitants per year (3).

It has been suggested that these assessments are imprecise and may considerably underestimate the true occurrence of the disorder $(1,4)$ given the results of prevalence evaluations of endogenous CS in pre-selected populations of patients with uncontrolled diabetes mellitus (DM) $(5,6,7)$ or hypertension (8). However, in these relatively small cohorts, the diagnostic criteria were not uniformly applied, and additional studies in patients

Published by Bioscientifica Ltd. 
with DM $(9,10,11)$ or obesity (12) yielded conflicting results. In one survey investigating the incidence of CS and Cushing's disease in commercially insured patients $<65$ years old in the United States, the authors reported a substantially higher incidence than previous estimates (13). However, they used a health insurance claims database that lacked granular clinical information to confirm the diagnosis. Furthermore, it was not clear if patients with exogenous CS were excluded (13).

The clinical presentation of CS is variable, with no single sign or symptom that is pathognomonic. According to the 2008 Endocrine Society guidelines, 24-h urinary free cortisol (UFC) measurement is one of the recommended tests for the diagnosis of CS (14). Values three to four times the upper limit of normal (ULN) are generally considered diagnostic $(1,4,15)$.

The aim of this study was to estimate the incidence of endogenous CS in a cohort of patients with significant hypercortisoluria and to analyze the performance of UFC screening.

\section{Methods}

The study was conducted at Maccabi Healthcare Services, the second largest publicly funded health maintenance organization (HMO) in Israel, and approved by its Ethics Review Board. Membership in one of the four HMOs in Israel is obligatory by law, and every resident of the country has the right to choose which of the four he/she prefers to join. Laboratory tests for urinary cortisol levels are covered by the HMOs. The computerized database of Maccabi Healthcare Services was reviewed for all individuals who underwent the UFC test from January 1, 2005 to December 31, 2014. Those with results that were twice the upper normal limit (UFC $>2 \times$ ULN) were identified, and their medical files were reviewed for a subsequent diagnosis of CS. Patients were included in the final study sample if they fulfilled the following two criteria:

1. A diagnosis of CS was established by an expert endocrinologist at the time of presentation according to the Endocrine Society clinical guidelines for the diagnosis of CS (14).

2. The diagnosis of CS was retrospectively ascertained by the study authors at the time of data collection according to the documented management and follow-up details. In patients who underwent surgery, CS was definitively diagnosed if the pathology report showed a pituitary ACTH-secreting adenoma/ ectopic ACTH-secreting tumor and/or if the clinical syndrome resolved after the operation along with the normalization of the laboratory findings. In patients who had not been operated by the time of data collection or who had not achieved remission after surgery, the diagnosis of CS was confirmed by the pathology report, if available, and/or the documentation of unequivocal biochemical parameters along with compatible clinical details regarding management. Patients were included only if repeated tests consistently yielded abnormal results until either the last follow-up or the administration of successful medical treatment or external radiation therapy. Abnormal results were defined as morning cortisol level $>1.8 \mu \mathrm{g} / \mathrm{dL}$ after an overnight $1 \mathrm{mg}$ dexamethasone suppression test (DST) and/or high late salivary cortisol measurements in addition to the elevated UFC tests ( $>2 \times$ ULN).

For each patient, the year in which the first elevated UFC result was recorded (2005-2014) was considered as the time of diagnosis.

Demographic and clinical parameters were collected by file review, as follows: age, sex, body mass index (BMI), diagnosis of hypertension, diabetes mellitus (DM) or impaired fasting glucose (IFG), and if specified, the reason for referral for the UFC test. Additionally, in patients subsequently diagnosed with CS, data were retrieved on the specific cause of the hypercortisolism and the clinical course after diagnosis. In patients who were not diagnosed with CS, the medical files were screened for potential causes for hypercortisolism and further cortisol level assessments.

Urine cortisol was measured using a commercial radioimmunoassay (DiaSorin, Saluggia, Italy or Siemens Healthcare Diagnostics).

\section{Statistical analysis}

On the basis of the present findings, we calculated the annual incidence of new cases of CS in 2005-2014 in the general population insured by the HMO (Maccabi Healthcare Services) using the official publicly published data of the Israel National Insurance Institute on the yearly number of individuals insured at each of the four HMOs in Israel (https://www.btl.gov.il/Publications/ survey/Documents/seker_271.pdf). Subjects diagnosed/ not diagnosed with CS were compared for age and BMI by independent-samples $t$-test and for rates of hypertension, DM and IFG, by chi-square test. Observed differences were 
Table 1 Number of patients insured at the HMO each year from 2005 through 2014* and number and rate of individuals who underwent UFC measurement during the same years.

\begin{tabular}{|c|c|c|c|}
\hline Year & $\begin{array}{l}\text { No. of HMO-insured } \\
\text { patients }\end{array}$ & $\begin{array}{l}\text { No. of individuals who } \\
\text { underwent UFC test }\end{array}$ & $\begin{array}{c}\text { Rate of patients referred for UFC tests } \\
\text { of the HMO-insured population }(\%)\end{array}$ \\
\hline 2005 & 1692700 & 1804 & 0.11 \\
\hline 2006 & 1723400 & 1835 & 0.11 \\
\hline 2007 & 1761600 & 2371 & 0.13 \\
\hline 2008 & 1813600 & 3000 & 0.17 \\
\hline 2009 & 1860100 & 4056 & 0.22 \\
\hline 2010 & 1902100 & 4747 & 0.25 \\
\hline 2011 & 1939200 & 5220 & 0.27 \\
\hline 2012 & 1975300 & 5535 & 0.28 \\
\hline 2013 & 2011774 & 6151 & 0.31 \\
\hline 2014 & 2050788 & 6464 & 0.32 \\
\hline
\end{tabular}

*According to official data of the Israel National Insurance Institute.

UFC, urinary free cortisol.

assumed to be statistically significant if the probability of chance occurrence ( $P$ value) was less than 0.05 .

\section{Results}

According to the Israel National Insurance Institute reports (https://www.btl.gov.il/Publications/survey/Documents/ seker_271.pdf), the number of individuals insured at Maccabi Healthcare Services in 2005 (first year of the study) was 1692700 and gradually increased by $21.2 \%$ to 2050788 in 2014 (last year of the study) (Table 1). In all the study years, the number of people insured at this HMO accounted for approximately $25 \%$ of the Israeli population (https://www.btl.gov.il/Publications/survey/ Documents/seker_271.pdf).

During the same years, UFC levels were measured in 41183 subjects at the Central Laboratory of Maccabi Healthcare Services. Of these, 34562 (83.9\%) had normal-range results. The number of subjects referred for UFC testing increased every year during the study period (Table 1). In 2005, UFC screening was performed in 1804 persons, and in 2014, in 6464 persons, for an increase of 2.9-fold in the rate of individuals who underwent the test (Table 1).

UFC levels $>2 \times$ ULN were found in 510 subjects (1.2\%), and UFC levels $>3 \times$ ULN, in 214 subjects $(0.5 \%)$.

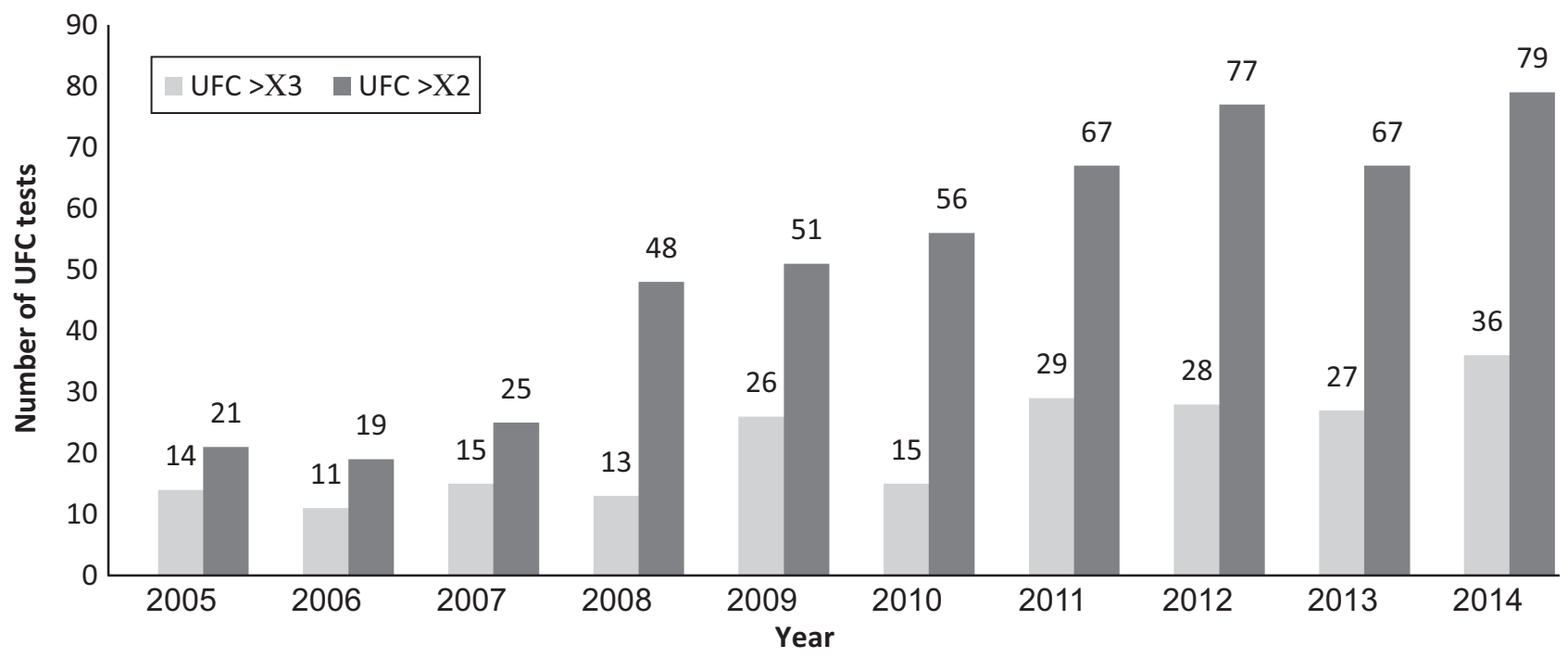

Figure 1

Distribution of 510 patients with UFC >2x ULN by year, from 2005 to 2014. (Ten patients first diagnosed before January 1, 2005 were excluded.) UFC, urinary free cortisol; ULN, upper limit of normal. 
Table 2 Classification of 85 patients with CS by specific diagnosis.

\begin{tabular}{lcc}
\hline Diagnosis & No. of patients (\%) \\
\hline Cushing's disease & $47(55.3)$ \\
Adrenal CS, total & $32(37.6)$ \\
Adrenal adenoma & $22(25.9)$ \\
Adrenocortical carcinoma & $6(7.1)$ \\
Bilateral adrenal mass & $4(4.7)$ \\
Ectopic ACTH secretion & $4(4.7)$ \\
Unknown & $2(2.4)$ \\
\hline
\end{tabular}

ACTH, adrenocorticotropic hormone; CS, Cushing's syndrome.

The number of cases of UFC $>2 \times$ ULN increased over time in most study years, in consonance with the increase in the total number of UFC tests performed. By contrast, the number of cases of UFC $>3 \times$ ULN remained quite stable during the periods from 2005 to 2008 and from 2009 to 2013 (Fig. 1).

Eighty-five subjects of the 510 subjects with UFC $>2 \times$ ULN (16.7\%) were subsequently diagnosed with CS. These patients constituted $0.2 \%$ of all individuals who underwent UFC testing during this 10-year period. Classification of the patients diagnosed with CS according to the specific cause of hypercortisolism is shown in Table 2. The most common diagnosis was Cushing's disease, in 47 patients (55.3\%), of whom 46 had undergone trans-sphenoidal surgery by the time of data collection. Remission was achieved in 42 patients, 4 of them after additional treatment (repeated transsphenoidal surgery in 2 patients; radiosurgery and bilateral adrenalectomy, 1 patient each). Recurrence was documented in 3 patients within 2-9 years after surgery. Of the 32 patients with adrenal CS, 26 underwent adrenalectomy, leading to remission in 23 . Two patients refused surgery and 2 were not operated because of bilateral adrenal masses and metastatic adrenocortical carcinoma; in 2 patients, no data on treatment were available. Four patients were diagnosed with CS due to ectopic ACTH secretion. One had a neuroendocrine carcinoma and was treated with chemotherapy, one underwent bilateral adrenalectomy and one was treated with ketoconazole after surgical excision of a lung carcinoid; in one patient, management data were unavailable. Overall, until the time of data collection, 74 patients with CS (87.1\%) had undergone surgery. Two patients refused surgery, and 5 patients were treated medically. In 4 patients, no full data on treatment were available.

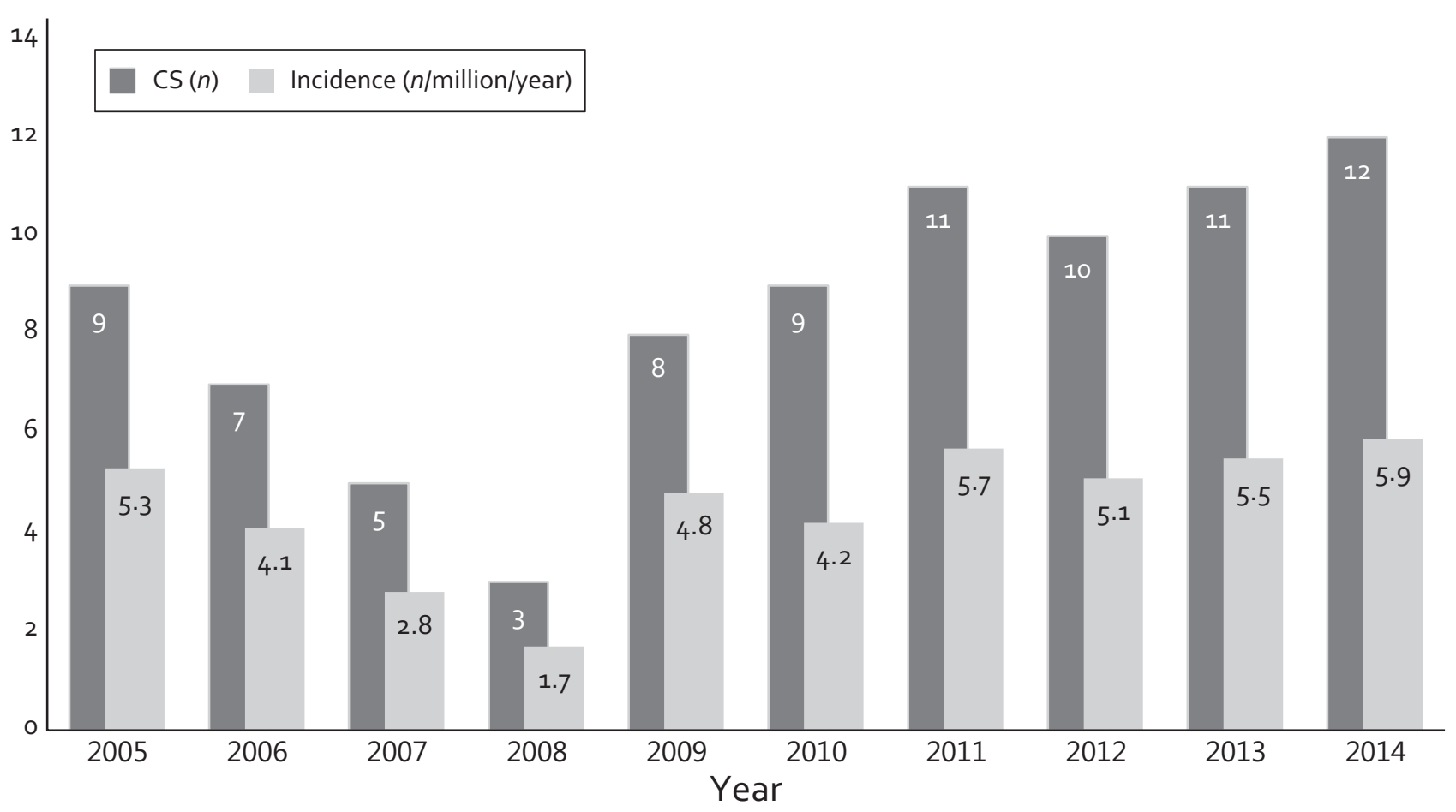

Figure 2

Distribution of 85 patients with CS diagnosed in 2005-2014 by number and incidence in the general HMO-insured population per year. CS, Cushing syndrome. 
Table 3 Characteristics of subjects with UFC $>2 \times$ ULN, with and without CS.

\begin{tabular}{|c|c|c|c|}
\hline & CS $(n=85)$ & No. CS $(n=425)$ & $P$ value \\
\hline Age (year), mean \pm SD (median) & $47.2 \pm 15.1(46)$ & $48.4 \pm 15.7(48)$ & NS \\
\hline Female, $n(\%)$ & $63(74.1)$ & $207(48.7)$ & 0.0001 \\
\hline BMI $\left(\mathrm{kg} / \mathrm{m}^{2}\right)$, mean $\pm \mathrm{SD}($ median $)$ & $30.3 \pm 6.4(30)$ & $35 \pm 9.8(35.2)$ & 0.0001 \\
\hline Hypertension, n (\%) & $51(60)$ & $225(52.9)$ & 0.03 \\
\hline Type 2 DM or IFG, $n(\%)$ & $39(45.9)$ & $209(49.2)$ & NS \\
\hline Hypertension + DM/IFG, $n(\%)$ & $30(35.3)$ & $137(32.2)$ & NS \\
\hline
\end{tabular}

CS, Cushing's syndrome; DM, diabetes mellitus; IFG, impaired fasting glucose; UFC, urinary free cortisol; ULN, upper limit of the normal range.

The absolute number of patients and the incidence of CS per year in the HMO-insured population are shown in Fig. 2. The mean annual incidence of CS was $4.5 \pm 1.4$ new cases/million/year (median 4.9/million/year, range 1.7-5.9/million/year).

Analysis of the maximal UFC levels in patients with CS yielded a mean value of $8.2 \pm 12.6 \times$ ULN (median $4.6 \times$ ULN, range $2.1-224 \times$ ULN). In 71 of the 85 patients with CS $(83.5 \%)$, the maximal UFC level was $>3 \times$ ULN. These patients constituted $33.2 \%$ of the 214 individuals with UFC level $>3 \times$ ULN. In only 14 patients $(16.5 \%)$ was the maximal UFC level 2-3× ULN.

A search of the medical files of the 425 patients with UFC levels $>2 \times$ ULN who were not diagnosed with CS revealed potential causes of hypercortisoluria in 159 . These included 105 patients who underwent urinary cortisol measurement while on exogenous glucocorticoid preparations and 44 patients who had documented conditions associated with hypercortisolism, namely, depression and other psychiatric conditions $(n=19)$, anorexia nervosa $(n=5)$, pregnancy $(n=1)$, alcohol dependence $(n=1)$, acute illness $(n=14)$ and sleep apnea with chronic hypoxia $(n=4)$. In the remaining 10 patients, the elevated UFC level was considered a laboratory error, and repeated UFC testing within 1 month revealed normal results.

Of the 266 patients with no apparent cause of the hypercortisolism, CS was formally excluded in 89 based on at least two of the following tests: repeated UFC screening showing normal-range levels; overnight $1 \mathrm{mg}$ DST showing a morning plasma cortisol level of $<1.8 \mu \mathrm{g} / \mathrm{dL}(50 \mathrm{nmol} / \mathrm{L}) ;$ and late-night salivary cortisol measurement showing normal laboratory-range levels. An additional 127 patients underwent only one further screening test: repeated UFC measurement in 45 patients, which showed normal levels in 33 and repeated high levels in 12; overnight $1 \mathrm{mg}$ DST in 74, showing cortisol $<1.8 \mu \mathrm{g} / \mathrm{dL}$ in all cases; and late-night salivary cortisol assessment in 8 , showing normal-range values in all cases. In 13 patients, we found only basal morning cortisol levels. In the remaining 37 patients, no further cortisol measurements were performed.

In 420 of the 510 patients with UFC $>2 \times$ ULN, the reason for referral for screening was specified in the medical file. The most common reason, in 246 patients (58.6\%), was obesity with/without other findings suspicious of CS. This subgroup included 148 patients who underwent the UFC test before planned bariatric surgery; only 2 were positive for CS. Fifty-nine of the 420 patients with a specified reason (14\%) were referred after detection of an adrenal mass, of whom 9 were consequently diagnosed with adrenal CS.

Table 3 compares the clinical characteristics of the tested subjects with and without a final diagnosis of CS. The CS group was predominantly female (74.1\%), and the non-CS group, predominantly male. In both groups, obesity, hypertension and DM were prevalent, but BMI was lower and hypertension was more common in the patients with CS.

\section{Discussion}

This study shows that during the 10-year period from 2005 to 2014, the number of UFC tests performed in a major HMO in Israel dramatically increased. However, there was no parallel increase in the detection rate of CS in patients with UFC $>2 \times$ ULN. On the basis of the results in the present cohort, we calculated a mean incidence of CS in the general HMO-insured population of 4.5 per million per year. This value is more than twice that reported in the more recent of the two general population-based surveys published in the medical literature (2). The incidence in the last four years of our survey (2011-2014) was even higher, namely, 5.1-5.9 new cases per million per year.

In their study of the incidence of CS, Lindholm et al. (2) identified patients using data from the National Danish Patient Register and departments of endocrinology and neurosurgery in medical centers in Denmark. In the absence of a national patient register in Israel, we used 
documented UFC screening measurements to retrieve a large proportion of the patients diagnosed with CS during the study period. The use of UFC tests to establish the diagnosis of CS was recognized already in the 1970s $(16,17)$. In 2008 , the Endocrine Society guidelines recommended the UFC test as one of three diagnostic screens for CS, along with overnight $1 \mathrm{mg}$ dexamethasone suppression and late-night salivary cortisol measurement, and suggested that CS cannot be diagnosed unless findings on at least two of the tests were abnormal (14). We assumed that, practically, the vast majority of patients with CS undergo UFC measurement at least once during the diagnostic process and that by using a cut point of UFC $>2 \times$ ULN, we would be able to detect most patients diagnosed with CS during the study years among the surveyed population $(1,4,15)$.

Although our study and that of Lindholm et al. (2) applied different practices to identify patients with CS, we speculate that this is not the reason for the higher incidence reported here. Indeed, the authors of the Danish survey expressed confidence that owing to their exhaustive search, they had included all patients with pituitary and adrenal CS in Denmark (2). Rather, we attribute the difference to the long time (two decades) that elapsed between the studies, such that the higher incidence in ours probably reflects the increasing tendency to diagnose CS at earlier, milder stages (18). The more extensive use of computed tomography and magnetic resonance imaging in recent years has led to a growing number of incidentally found adrenal masses, some of which turn out to be cortisol-producing adenomas (19). The prevalence of 'adrenal incidentalomas' increases with age, up to a rate of $7 \%(19,20,21)$, with $6-23 \%$ of affected patients found to have 'subclinical' or subtle CS $(19,22$, 23). Accordingly, in Israel, a retrospective case series of 100 consecutive patients with adrenal incidentalomas found that $8 \%$ had subclinical or overt CS (24). In the present cohort, 9 of the 32 patients (28.1\%) with adrenal CS were referred for UFC screening because of the detection of an adrenal mass. A diagnosis of adrenal CS was established in $37.6 \%$ of the total patients, compared with $28.9 \%$ in the survey of Lindholm et al. (2). This rate of non-ACTHdependent CS is higher than expected according to previous reports $(1,4)$.

A remarkable finding in this study is the disproportionate increase over time in the number of patients referred for UFC testing relative to the growth of the surveyed population and the rate of CS diagnosis. This discrepancy is most likely explained by an increase in the prevalence of CS screening among patients with obesity, type $2 \mathrm{DM}$ or hypertension in the absence of more specific features of CS. The sharp growth in CS screening may also be associated with the rapid upsurge in the performance of bariatric surgery in recent years. According to the data of the Israel National Hospital Discharge Register, the number of bariatric procedures increased during the study years from 1390 in 2005 to 8841 in 2014 (http://www. health.gov.il/PublicationsFiles/bariatric_2014.pdf). In the absence of standardized endocrine assessment protocol before bariatric surgery, the preoperative referral of obese patients for UFC testing is common in Israel (D.H., personal communication).

The screening strategy proposed by the Endocrine Society guidelines is intended to reduce the number of false-positive test results. Therefore, widespread testing for CS in the absence of specific features is discouraged, and a case-finding approach is preferred (14). A recent literature review showed that in large series, only $\leq 1 \%$ of patients with obesity, type $2 \mathrm{DM}$ or hypertension were diagnosed with CS by routine screening of individuals (18). Nevertheless, in this study, the most common reason specified for UFC measurement was obesity. Of the 246 subjects with this indication, 148 (60.2\%) were referred before planned bariatric surgery; only 2 of them were subsequently diagnosed with CS. These findings are in accordance with two series including 783 and 609 consecutive morbidly obese patients who underwent the overnight $1 \mathrm{mg}$ DST, of whom $0.8 \%$ and $0.6 \%$ respectively, were found to have CS $(25,26)$.

We postulate that obesity was also a main reason for UFC testing in the individuals with normal-range results, as circulating cortisol concentrations are usually normal (or even slightly reduced) in obesity (27). Baid et al. (12), in a study of the specificity of screening tests for CS in an overweight and obese population, found that none of the 369 patients had CS although they had a mean of 5-6 signs or symptoms. However, severe obesity itself can sometimes raise UFC levels, leading to false-positive screening results (14). Accordingly, we found that the median BMI of the patients with CS was within the limits of overweight, whereas the median BMI of the patients without CS was compatible with severe obesity.

Not surprisingly, the vast majority of subjects referred for UFC screening in our study had normal-range results. Most of those diagnosed with CS had levels of $3 \times$ ULN, and only 14 patients (16.5\%) had a maximal level of $2-3 \times$ ULN. This finding is compatible with other studies showing that patients with CS usually have UFC values at least threefold higher than the $\operatorname{ULN}(1,4,15)$. In this study, urine cortisol measurements were obtained using 
antibody-based immunoassays, which can be affected by cross-reactivity with cortisol metabolites and synthetic glucocorticoids (28). Indeed, the most common cause of possibly false-positive UFC results in this study was the use of an exogenous glucocorticoid preparation. Immunologic tests are still in widespread use, although they are gradually being replaced by structurally based assays, such as high-performance liquid chromatography (HPLC) and tandem mass spectrometry (LC-MS/MS), which do not pose a cross-reactivity problem $(29,30,31$, 32). It may be assumed that had we tested the patients with HPLC or LC-MS/MS, the percentage of those with normal UFC tests would have been even higher.

The strengths of this study are the probable inclusion of a large proportion of the patients diagnosed with CS in the surveyed population during the study years and the availability of detailed clinical and laboratory data for the included individuals.

A major limitation of our study is that the cohort of patients with UFC $>2 \times$ ULN was not fully representative of all HMO-insured patients who were screened for CS during the study years. Thus, the calculated annual incidence of CS in the population insured by the $\mathrm{HMO}$, based only on the patients identified in this study, is almost certainly an underestimation. There were undoubtedly some patients who were referred for other screening tests owing to personal or physician preference or situations in which the UFC test is inappropriate (e.g., moderate-to-severe renal impairment). Others might have been diagnosed with lower than twofold elevations of UFC levels. In a study on UFC levels in 152 patients with Cushing's disease, $17.1 \%$ had UFC levels between $1.5 \times$ and $2.0 \times$ ULN (33). Some patients with cyclic or mild CS may even present with normal UFC values (34). Furthermore, some of the 425 patients with hypercortisoluria who were included in our cohort but were not diagnosed with CS could have been wrongly excluded due to incomplete evaluation of their cortisol levels. Thus, although our findings reveal that the incidence of CS is higher than previously reported, the true incidence is likely even higher than that reported here.

In summary, an increasing number of individuals are being referred for UFC measurement, apparently with no justification. Specifically, the expected yield of routine UFC testing in patients scheduled for bariatric surgery is low, and guidelines regarding the need for endocrine evaluation in these patients are warranted. Although the design of this study is not appropriate for a reliable and precise calculation of the annual incidence of CS in the general population, it may be concluded that the incidence is considerably higher than that previously suggested.

\section{Declaration of interest}

The authors declare that there is no conflict of interest that could be perceived as prejudicing the impartiality of the research reported.

\section{Funding}

This research did not receive any specific grant from any funding agency in the public, commercial, or not-for-profit sector.

\section{References}

1 Newell-Price J, Bertagna X, Grossman AB \& Nieman LK. Cushing's syndrome. Lancet 2006367 1605-1617. (doi:10.1016/S01406736(06)68699-6)

2 Lindholm J, Juul S, Jørgenson JO, Astrup J, Bjerre P, FeldtRasmussen U, Hagen C, Jørgensen J, Kosteljanetz M, Kristensen L et al. Incidence and late prognosis of Cushing's syndrome: a populationbased study. Journal of Clinical Endocrinology and Metabolism 200186 117-123. (doi:10.1210/jc.86.1.117)

3 Etxabe J \& Vazquez JA. Morbidity and mortality in Cushing's disease: an epidemiological approach. Clinical Endocrinology 199440 479-484. (doi:10.1111/j.1365-2265.1994.tb02486.x)

4 Susmeeta T, Sharma ST, Nieman LK \& Feelders RA. Cushing's syndrome: epidemiology and developments in disease management. Clinical Epidemiology 20157 281-293. (doi:10.2147/clep.s44336)

5 Leibowitz G, Tsur A, Chayen SD, Salameh M, Raz I, Cerasi E \& Gross DJ. Pre-clinical Cushing's syndrome: an unexpected frequent cause of poor glycaemic control in obese diabetic patients. Clinical Endocrinology 199644 717-722. (doi:10.1046/j.13652265.1996.737558.x)

6 Catargi B, Rigalleau V, Poussin A, Ronci-Chaix N, Bex V, Vergnot V, Gin H, Roger P \& Tabarin A. Occult Cushing's syndrome in type-2 diabetes. Journal of Clinical Endocrinology and Metabolism $2003 \mathbf{8 8}$ 5808-5813. (doi:10.1210/jc.2003-030254)

7 Terzolo M, Reimondo G, Chiodini I, Castello R, Giordano R, Ciccarelli E, Limone P, Crivellaro C, Martinelli I, Montini M et al. Screening of Cushing's syndrome in outpatients with type 2 diabetes: results of a prospective multicentric study in Italy. Journal of Clinical Endocrinology and Metabolism 201297 3467-3475. (doi:10.1210/ jc.2012-1323)

8 Omura M, Saito J, Yamaguchi K, Kakuta Y \& Nishikawa T. Prospective study on the prevalence of secondary hypertension among hypertensive patients visiting a general outpatient clinic in Japan. Hypertension Research 200427 193-202. (doi:10.1291/hypres.27.193)

9 Mullan K, Black N, Thiraviaraj A, Bell PM, Burgess C, Hunter SJ, McCance DR, Leslie $\mathrm{H}$, Sheridan B \& Atkinson AB. Is there value in routine screening for Cushing's syndrome in patients with diabetes? Journal of Clinical Endocrinology and Metabolism 201095 2262-2265. (doi:10.1210/jc.2009-2453)

10 Reimondo G, Pia A, Allasino B, Tassone F, Bovio S, Borretta G, Angeli A \& Terzolo M. Screening of Cushing's syndrome in adult patients with newly diagnosed diabetes mellitus. Clinical Endocrinology 200767 225-229. (doi:10.1111/j.1365-2265.2007.02865.x)

11 Newsome S, Chen K, Hoang J, Wilson JD, Potter JM \& Hickman PE. Cushing's syndrome in a clinic population with diabetes. Internal Medicine Journal 200838 178-182. (doi:10.1111/j.14455994.2007.01434.x)

12 Baid SK, Rubino D, Sinaii N, Ramsey S, Frank A \& Nieman LK. Specificity of screening tests for Cushing's syndrome in an overweight 
and obese population. Journal of Clinical Endocrinology and Metabolism 200994 3857-3864. (doi:10.1210/jc.2008-2766)

13 Broder MS, Neary MP, Chang E, Cherepanov D \& Ludlam WH. Incidence of Cushing's syndrome and Cushing's disease in commercially-insured patients $<65$ years old in the United States. Pituitary 201518 283-289. (doi:10.1007/s11102-014-0569-6)

14 Nieman LK, Biller BM, Findling JW, Newell-Price J, Savage MO, Stewart PM \& Montori VM. The diagnosis of Cushing's syndrome: an Endocrine Society Clinical Practice Guideline. Journal of Clinical Endocrinology and Metabolism 200893 1526-1540. (doi:10.1210/ jc.2008-0125)

15 Newell-Price J, Trainer P, Besser M \& Grossman A. The diagnosis and differential diagnosis of Cushing's syndrome and pseudo-Cushing's states. Endocrine Reviews 199819 647-672. (doi:10.1210/er.19.5.647)

16 Crapo L. Cushing's syndrome: a review of diagnostic tests. Metabolism 197928 955-977. (doi:10.1016/0026-0495(79)90097-0)

17 Melby JC. Assessment of adrenocortical function. New England Journal of Medicine 1971285 735-739. (doi:10.1056/NEJM197109232851306)

18 Shimon I. Screening for Cushing's syndrome: is it worthwhile? Pituitary 201518 201-205. (doi:10.1007/s11102-015-0634-9)

19 Nieman LK. Approach to the patient with an adrenal incidentaloma. Journal of Clinical Endocrinology and Metabolism 201095 4106-4113. (doi:10.1210/jc.2010-0457)

20 Herrera MF, Grant CS, van Heerden JA, Sheedy PF \& Ilstrup DM. Incidentally discovered adrenal tumors: an institutional perspective. Surgery 1991110 1014-1021.

21 Bovio S, Cataldi A, Reimondo G, Sperone P, Novello S, Berruti A, Borasio P, Fava C, Dogliotti L, Scagliotti GV et al. Prevalence of adrenal incidentaloma in a contemporary computerized tomography series. Journal of Endocrinology Investigation 200629 298-302. (doi:10.1007/BF03344099)

22 Olsen H, Nordenström E, Bergenfelz A, Nyman U, Valdemarsson S \& Palmqvist E. Subclinical hypercortisolism and CT appearance in adrenal incidentalomas: a multicenter study from Southern Sweden. Endocrine 201242 164-173. (doi:10.1007/s12020-012-9622-2)

23 Mantero F, Terzolo M, Arnaldi G, Osella G, Masini AM, Alì A, Giovagnetti M, Opocher G \& Angeli A. A survey on adrenal incidentaloma in Italy. Study Group on Adrenal Tumors of the Italian Society of Endocrinology. Journal of Clinical Endocrinology and Metabolism 200085 637-644. (doi:10.1210/jc.85.2.637)

24 Tsvetov G, Shimon I \& Benbassat C. Adrenal incidentaloma: clinical characteristics and comparison between patients with and without extra adrenal malignancy. Journal of Endocrinology Investigation 2007 30 647-652. (doi:10.1007/BF03347444)
25 Fierabracci P, Pinchera A, Martinelli S, Scartabelli G, Salvetti G, Giannetti M, Pucci A, Galli G, Ricco I, Querci G et al. Prevalence of endocrine diseases in morbidly obese patients scheduled for bariatric surgery: beyond diabetes. Obesity Surgery 201121 54-60. (doi:10.1007/s11695-010-0297-6)

26 Janković D, Wolf P, Anderwald CH, Winhofer Y, Promintzer-Schifferl M, Hofer A, Langer F, Prager G, Ludvik B, Gessl A et al. Prevalence of endocrine disorders in morbidly obese patients and the effects of bariatric surgery on endocrine and metabolic parameters. Obesity Surgery 201222 62-69. (doi:10.1007/ s11695-011-0545-4)

27 Pecori Giraldi F, Ambrogio AG, De Martin M, Fatti LM, Scacchi M \& Cavagnini F. Specificity of first-line tests for the diagnosis of Cushing's syndrome: assessment in a large series. Journal of Clinical Endocrinology and Metabolism 200792 4123-4129. (doi:10.1210/ jc.2007-0596)

28 Turpeinen U \& Hämäläinen E. Determination of cortisol in serum, saliva and urine. Best Practice and Research Clinical Endocrinology and Metabolism 201327 795-801. (doi:10.1016/j.beem.2013.10.008)

29 Canalis E, Reardon GE \& Caldarella M. A more specific, liquidchromatographic method for free cortisol in urine. Clinical Chemistry 198228 2418-2420.

30 Turpeinen U, Markkanen H, Välimäki M \& Stenman UH. Determination of urinary free cortisol by HPLC. Clinical Chemistry 199743 1386-1391.

31 Turpeinen U \& Stenman U-H. Determination of urinary free cortisol by liquid chromatography-tandem mass spectrometry. Scandinavian Journal of Clinical Laboratory Investigation 200363 143-150. (doi:10.1080/00365510310000097)

32 Wood L, Ducroq DH, Fraser HL, Gillingwater S, Evans C, Pickett AJ, Rees DW, John R \& Turkes A. Measurement of urinary free cortisol by tandem mass spectrometry and comparison with results obtained by gas chromatography-mass spectrometry and two commercial immunoassays. Annals of Clinical Biochemistry 200845 380-388. (doi:10.1258/acb.2007.007119)

33 Petersenn S, Newell-Price J, Findling JW, Gu F, Maldonado M, Sen K, Salgado LR, Colao A, Biller BM \& Pasireotide B2305 Study Group. High variability in baseline urinary free cortisol values in patients with Cushing's disease. Clinical Endocrinology 201480 261-269. (doi:10.1111/cen.12259)

34 Kidambi S, Raff H \& Findling JW. Limitations of nocturnal salivary cortisol and urine free cortisol in the diagnosis of mild Cushing's syndrome. European Journal of Endocrinology 2007157 725-731. (doi:10.1530/EJE-07-0424)

Received 29 July 2016

Revised version received 27 September 2016

Accepted 13 October 2016 